The United States Government retains and the publisher, by accepting the article for publication, acknowledges that the United States Government retains a nonexclusive, paid-up, irrevocable, world-wide license to publish or reproduce the published form of this manuscript, or allow others to do so, for United States Government purposes. The Department of Energy will provide public access to these results of federally sponsored research in accordance with the DOE Public Access Plan (http://energy.gov/downloads/doe-public-access-plan).

(C) 2017. This manuscript version is made available under the Elsevier user license http://www.elsevier.com/open-access/userlicense/1.0/ 


\title{
An automated analysis workflow for optimization of force-field parameters using neutron scattering data
}

Vickie E. Lynch ${ }^{\mathrm{a}}$, Jose M. Borreguero ${ }^{\mathrm{a}}$, Debsindhu Bhowmik ${ }^{\mathrm{c}}$, Panchapakesan Ganesh $^{\text {b,c }}$, Bobby G. Sumpter ${ }^{\text {b,c }}$, Thomas E. Proffen ${ }^{\mathrm{a}}$, Monojoy Goswami ${ }^{\mathrm{b}, \mathrm{c}, *}$

\author{
Oak Ridge National Laboratory, Oak Ridge, TN, 37831 \\ ${ }^{a}$ Neutron Data Analysis \& Visualization Division \\ ${ }^{b}$ Center for Nanophase Material Sciences \\ ${ }^{c}$ Computational Sciences \& Engineering Division
}

\begin{abstract}
Large-scale simulations and data analysis are often required to explain neutron scattering experiments to establish a connection between the fundamental physics at the nanoscale and data probed by neutrons. However, to perform simulations at experimental conditions it is critical to use correct force-field (FF) parameters which are unfortunately not available for most complex experimental systems. In this work, we have developed a workflow optimization technique to provide optimized FF parameters by comparing molecular dynamics (MD) to neutron scattering data. We describe the workflow in detail by using an example system consisting of tRNA and hydrophilic nanodiamonds in a deuterated water $\left(\mathrm{D}_{2} \mathrm{O}\right)$ environment. Quasi-elastic neutron scattering (QENS) data show a faster motion of the tRNA in the presence of nanodiamond than without the ND. To compare the QENS and MD results quantitatively, a proper choice of FF parameters is necessary. We use an efficient workflow to optimize the FF parameters between the hydrophilic nanodiamond and water by comparing to the QENS data. Our results show that we can obtain accurate FF parameters by using this technique. The workflow can be generalized to other types of neutron data for FF optimization, such as vibrational spectroscopy and spin echo.
\end{abstract}

Keywords: force-field optimization, molecular dynamics,

\footnotetext{
*Corresponding author

Email address: goswamimeornl . gov (Monojoy Goswami)
} 
neutron data analysis, quasi-elastic neutron scattering,

biopolymers, CHARMM

\section{Introduction}

The basis of neutron scattering experiments is the subatomic particle neutron. Unlike protons and electrons which have intrinsic charges, neutrons do not have an intrinsic net charge. This distinction is prominent in neutron-matter interactions compared to proton-matter or electron-matter interactions which are dictated by long-range electrostatic forces. The electrostatic interactions between protons/electrons with material hinder the motion of these subatomic particles whereas the neutrons are rarely affected. This enables the neutrons an ability to penetrate deeper into the material. This property of neutrons is regularly harnessed to understand the bulk properties of materials in neutron scattering experiments. Therefore, neutron scattering is a prominent and versatile technique to understand structure and dynamics at molecular length scales.

A large number of scattering experiments are carried out every year at the Spallation Neutron Source (SNS), Oak Ridge National Laboratory (ORNL) [1] by users across the world. In majority of these cases, the data require further analysis to delve deeper into a fundamental understanding of the material properties. Often it is found that the collected neutron data cannot uniquely resolve the structural or dynamical properties of the sample without a set of complementary simulations. For such cases, it becomes necessary to perform large-scale simulations to match the experimental length and time scales. However, appropriate values for all the simulation parameters are not always available, especially force-field (FF) parameters for complex systems such as, polymers with complex architecture and biological macromolecules. In these situations, simulations are performed by approximating FF parameters derived from ab initio calculations or from comparison to other experimental techniques probing physical quantities uncorrelated to the dynamics probed by neutrons. The resulting simulations offer, at best, a qualitative explanation of the observed neutron data [2, 3].

Designing simulation systems in parallel with the experimental system is a classic endeavor for the soft matter simulation community. Because of the complex nature of 
the interactions between different species of the macromolecules with the environment, the FF parameters are sometimes 'educated guesses' that may or may not complement the experiments quantitatively [4, 5]. Hence it becomes necessary to develop newer simulation techniques or obtain 'exact' FF parameters. In order to achieve quantitative consistency between simulations and experiments, we have developed a workflow that performs large-scale simulations, calculates the relevant scattering function, compares the results to neutron experimental data and iterates the FF parameters until a value of the FF is optimized to experimental accuracy. We integrated existing codes at SNS to develop a complimentary automated system that will solve common optimization problems for a widespread set of simulation systems and neutron experiments.

The robustness of our computational approach can be manifested by applying the optimization technique to a test problem. Moreover, an ideal test system should contain both neutron experiment and computer simulation data. Such an ideal system was available to us. We used QENS data of a three component system made of transferRNA (tRNA), water $\left(\mathrm{D}_{2} \mathrm{O}\right)$ and nanodiamond (ND). The QENS data for the tRNA dynamics were derived on the BASIS instrument [6]. It was found that the dynamics of tRNA is faster in the presence of nanodiamond (ND) than in the absence of it. To gain a fundamental understanding of the physics behind the enhanced dynamics in ND surface, we performed MD simulation for hydrated tRNA with and without ND. The QENS results were at first counterintuitive, given that an obstruction (in this case ND) causes the large tRNA molecule to move faster compared to a freestanding tRNA. The faster dynamics of tRNA on the ND surface might be the result of a different kind of interaction between ND with water and tRNA. The ND surface is hydrophilic therefore the C-O interaction can explain the unexpected faster dynamics of tRNA on ND surface. Using our MD simulation, we observed that on the ND surface the tRNA molecules show weaker dynamic heterogeneity than the freestanding tRNA [7]. It is well established that weaker dynamic heterogeneity causes loosely bound molecules that results into faster tRNA dynamics in the ND presence. We also observed that the tRNA molecules swell in the presence of the ND. The swelled tRNA molecules moves faster and higher water uptake to the tRNA works as a 'plasticizer' or 'lubricant' as a result the tRNA molecules move faster. The fundamental physics understanding of 
the dynamics of tRNA with or without the presence of ND is reported earlier [7]. As the present manuscript deals with the computational method to optimize the forcefield parameters only, we did not discuss the detail of the fundamental findings in this manuscript. Rather we choose one particular system, the three-component system, data and used our workflow to optimize the force field parameter.

For the three component system, the 'exact' force-field parameters for the interactions between all the atoms were available except one. The attractive interactions of the hydrophilic ND surface were not known. Hydrophilic interactions in MD simulations can be modeled by two methods, $(i)$ explicit Coulomb interactions coming from each charge sites and, (ii) attractive LJ interactions between the hydrophilic molecules. While the first method closely resembles the experiments containing functional materials like functionalized ND, it is computationally expensive and often complex. Therefore, it is reasonable to use the second method for simulating large system like the present one. Moreover, the complex nature of ND surface requires careful simulation setup since the ND surfaces are expected to be hydrophilic overall, but have mixed terminations (both purely hydrophilic terminations such as - $\mathrm{OH}$ and purely hydrophobic terminations such as $-\mathrm{H}$ ). Therefore, we chose $\mathrm{LJ}$ interactions, that is in-build in our force-field, to mimic this condition in our simulation. In a previous work by Sendner et al. [8] it was shown that one can also vary the degree of hydrophobicity/hydrophilicity by varying the interaction energy $\left(\epsilon_{C O}\right)$ between carbon and oxygen atoms. When $\epsilon_{C O}$ was tuned between $-0.026 \mathrm{Kcal} / \mathrm{mol}$ and $-0.171 \mathrm{Kcal} / \mathrm{mol}$, the behavior of the ND surface changed from being hydrophobic to hydrophilic. In our simulation we chose $\epsilon_{C O}=-0.138 \mathrm{Kcal} / \mathrm{mol}$, to model an ND surface that is complex, but has a tendency to be overall hydrophilic. For carbonaceous materials, the interaction energies are typically observed in the same range, eg., for graphene-phospholipid interactions, the interaction energy ranges in the range of $100 \mathrm{cal} / \mathrm{mol}[9]$.

The preliminary MD simulations showed scattering profiles qualitatively equivalent to the QENS profiles, but a quantitative comparison was lacking. The discrepancy between experiment and simulation was the result of the assumed FF parameter between ND and water. In this work, we have used the optimization workflow to not only match the simulation with neutron experiment quantitatively, but to determine 
the nature (value) of the interaction between ND and water that eventually helped us understand the dynamics of the tRNA on ND surfaces.

The article is organized as follows: in Section 2 we describe the data collected in a QENS experiment and the procedure to calculate in silico counterparts from the MD simulations. In Section 3 we describe the computational details of the workflow, followed by an example of the performance of the workflow in Section 4 We end the article summarizing the important aspects of the workflow and future directions in developing simulation techniques for neutron data analysis.

\section{Optimization Procedure using QENS data}

In a typical QENS experiment, the sample to study is subjected to a constant flux of neutrons. The thickness of the sample is selected such that about $10 \%$ of the neutrons interact with atoms of the sample and are scattered in all directions, changing their momentum and energy. QENS is a limiting case of inelastic neutron scattering where the energy transfer, $E$, of the scattered neutron is small compared to its incident kinetic energy. These neutrons are detected and their change in direction and speed is logged. A histogram is generated providing a neutron count for every change in direction and speed or as in more customary, changes in neutron momentum, $Q$, and energy, $E$. The measured quantity (histogram), $S(Q, E)$, is referred to as the dynamic structure factor [10].

The connection between the incoherent part of the dynamic structure factor and self-diffusion theory [11] allows us to compute a closely related quantity from the atomic trajectories generated by MD simulations, namely the self-incoherent intermediate structure factor.

$$
I(Q, t)=\left\langle\frac{1}{N_{a t}} \sum_{i=1}^{N_{a t}}\left(b_{i}^{i n c}\right)^{2} e^{i \vec{Q}\left[\vec{r}_{i}\left(t_{0}+t\right)-\vec{r}_{i}\left(t_{0}\right)\right]}\right\rangle_{\Omega_{\vec{Q}}, t_{0}}
$$

where $N_{a t}$ is the number of atoms in the simulation, $b_{i}^{i n c}$ is the incoherent scattering length of atom $i$, and $\vec{r}_{i}(t)$ is the position of atom $i$ at time $t$. The angle bracket denotes the average over different initial time points $t_{0}$ and all possible orientations of the momentum transfer vector $\vec{Q}$. It is important to note that the experimentally 
measured QENS structure factor includes contributions from the coherent scattering within the sample. However, due to the large value of the incoherent scattering length of hydrogen, coherent scattering intensities are relatively smaller than the incoherent scattering intensities. For the simulation system of 8 nanodiamonds, ratios of coherent to incoherent scattering approximately range from $4 \%$ to $2 \%$ with an increase in momentum transfer, $Q$, from the lowest to the highest measured values. Hence, our fitting procedure takes only the incoherent part of the scattering into account.

A Fourier transform in time for $I(Q, t)$ and inclusion of the detailed balance correction [12] yields our structure factor.

$$
S_{s i m}(Q, E)=e^{\left(\frac{E}{2 k_{b} T}\right)} \frac{1}{\hbar} \int_{-\infty}^{\infty} e^{-i \frac{E t}{\hbar}} I(Q, t) d t
$$

A quantitative comparison between experimental $S_{\text {exp }}$ and simulated $S_{s i m}$ is only feasible if experimental uncertainties and coherent contributions from the sample are incorporated into the model. These are: (i) a background noise; (ii) additional intensity with no energy transfer $(E \equiv 0)$, originating from neutrons scattered by other sources than the sample as well as coherent scattering from the sample that is ignored in our numerical model; and (iii) errors when assigning particular values of energy and momentum transfer for each detected neutron. Insertion of these uncertainties result in the following expression for the model structure factor:

$$
S_{\text {model }}(Q, E)=R(Q, E) \otimes\left[\alpha \delta(E)+\beta S_{\text {sim }}(Q, E)\right]+(a E+b)
$$

where $a E+b$ models the linear background noise, typical of neutron detection devices, $\delta(E)$ models the additional intensity at $E \equiv 0$, and $R(Q, E)$ is a broadening function implementing inaccurate assignment in $Q$ and $E$. The function $R(Q, E)$, known as the resolution function, is the experimental structure factor collected at very low temperatures, typically below $15 \mathrm{~K}$. The resolution function is then convoluted to broaden the $\delta(E)$ and $S_{\text {sim }}$ intensities. Constants $\alpha, \beta, a$, and $b$ are free parameters in our optimization technique that compares $S_{\text {model }}$ and $S_{\text {exp }}$. There is an additional free parameter, the FF parameter that is the most important parameter in our optimization technique. While free parameters $\alpha, \beta, a$ and $b$ can be considered as parameters accounting for sample size, holding canister and features of the experimental instrument, the FF pa- 
rameter is intrinsic to the material under study. Changes in the FF parameter will affect implicitly on the force-field parameter. Optimal values are obtained from iterative minimization of the object function, usually the goodness-of-fit, $\chi^{2}$ given by,

$$
\chi^{2}=\sum_{i, j}\left(\frac{S_{e x p}\left(Q_{i}, E_{j}\right)-S_{\text {model }}\left(Q_{i}, E_{j}\right)}{\sigma\left(Q_{i}, E_{j}\right)}\right)^{2}
$$

where $\sigma(Q, E)$ is the experimental error associated to intensities $S_{\exp }(Q, E)$.

\section{Computational Method}

150 can be run in parallel, thereby significantly reducing the time it takes to optimize the FF parameter. Before starting the production runs, equilibration for each of the systems is achieved with a relatively short simulation. Each simulation must be submitted 
separately or combined in an ensemble run which requires a large number of computer cores, although this option may result in idle cores for simulations finishing earlier than the rest. The same may also apply for the subsequent runs to calculate the QENS intensities. In a 'manual mode', the user can prevent the waste of resources only by managing the set of batch jobs so that every job runs once the input data becomes available.

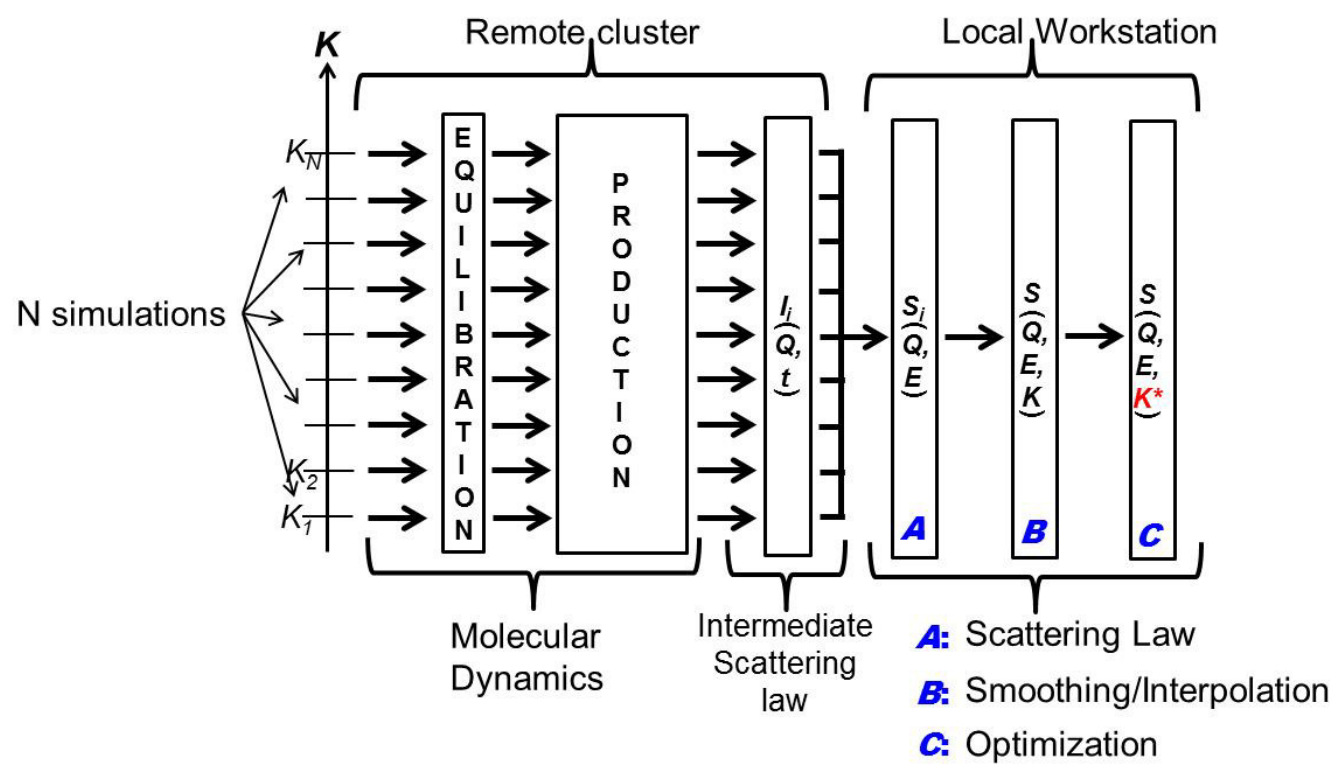

Figure 1: Workflow diagram for the generation of simulated QENS intensities. The workflow is carried out both using a remote HPC and a local workstation

The workflow shown in Fig 1 conveniently replaces the tedious management tasks by converting the manual operation to an automated mode. It saves valuable user time and is less prone to errors by avoiding excessive human intervention. The Pegasus [17] workflow, shown in Fig. 2 is created to implement the workflow diagram of Fig 1 . This workflow uses template files for NAMD configurations, Sassena input and FF parameters. A workflow configuration file contains the parameter values to be scanned. The workflow eventually incorporates the actual input files from the templates. The python daxgen [13, 17] program generates the workflow object into a set of template files using a python string formatter. Thereafter, the workflow is run using Pegasus commands. 
Pegasus uses Condor [20] and Globus for remote job submission to supercomputers. Therefore, it requires remote computer authentication keys or a grid certificate. No batch script is required for this process and the number of cores and time limit for each type job can be adjusted in the main configuration file.

We used the NAMD [14] simulation package which accepts various FF formats such as AMBER [21], CHARMM [22] and X-PLOR [23]. NAMD is highly efficient when running in parallel and has multiple environments available such as thermodynamic ensembles and temperature that can be tuned to mimic a variety of sample environments. Complex dynamic schemes available include restraints, replicas, and free energy calculations. In our example simulation, described in the next section, we have used the CHARMM FF, therefore the CHARMM structure file, coordinates, velocities and FF parameters are input to the NAMD simulation package. After each successful run, due to the drift of the simulation box, the global translation and rotation are removed from its output trajectory file using the AMBER15 [24] tool. The subtraction of global motion is necessary to remove the diffusive motions of the whole simulation box which have no counterpart in the experiments.

To calculate neutron scattering intensities from the MD trajectories, we use the Sassena code developed by Lindner et al. [15]. Sassena is a scalable parallel code for calculating the incoherent or coherent intermediate dynamic structure factors. The collective motion represented by coherent scattering and self-motion of the atoms represented by the incoherent scattering are two major scattering intensities obtained from neutron experiments, although only the incoherent part is relevant for QENS experiments. Sassena scales linearly up until 7000 cores for millions of atoms without restrictions. The resulting trajectory file generated by NAMD and the atom selection (hydrogen atoms in the case of QENS intensities) are input to Sassena to calculate the incoherent intensities only. The list of $Q$ values sampled in experiment and the accuracy of the orientational average on $Q$-space are also input to Sassena. The output of Sassena provides the simulated QENS intensities without the uncertainties endogenous to the experimental instrument as in eq2.

Once all the jobs from the workflow are completed, the optimization of the FF parameter is performed at a local workstation. We use Mantid [16] developed by Arnold 


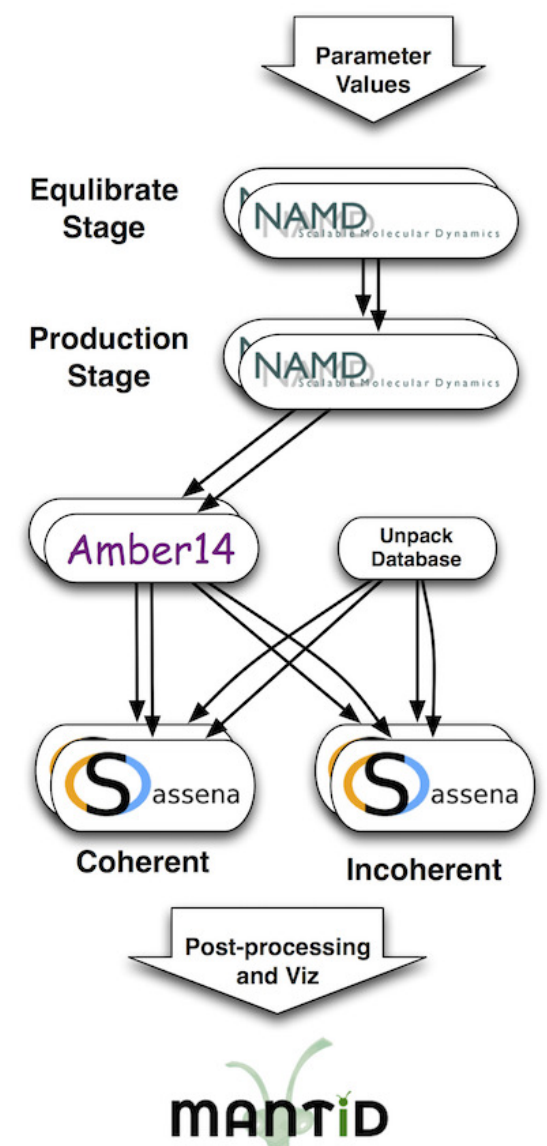

Figure 2: Pegasus SNS workflow for the optimization of the force-field parameters

et al. for neutron scattering data analysis to optimize the parameters by comparing $S_{\text {model }}(Q, E)$ of eq 3 with the experimental $S_{\text {expt }}(Q, E)$, as described in Section 2 . Since MD simulations are finite in time and size, the scattering function derived from the simulation has errors leading to local minima in the objective function, $\chi^{2}$. We solved this issue by interpolating the dynamic structure factor, $S_{\text {sim }}(Q, E)$ shown in eq 2 ,with a cubic spline interpolator, $\bar{S}(Q, E, f)$, along an extra parameter $f$. The parameter, $f$, can be any environment variable such as temperature or a slightly inaccurate FF parameter, as in our example case described in Section 4, that needs to be optimized. The example case and the choice of the particular parameter will be dis- 
cussed in Section 4 in detail. The above technique is already implemented in Mantid package [16].

A systematic approach is required to perform the complex optimization process. First we load the $S_{\text {expt }}(Q, E)$ data and the resolution function. $S_{\text {expt }}(Q, E)$ data is a function of momentum transfer, $Q$ (in $\AA^{-1}$ ) and energy transfer, $E$ (in $m e V$ ). The resolution function is the $S(Q, E)$ of the sample at low temperature. $I(Q, t)$ of the NAMD simulation data (eq 1) were obtained from Sassena output (HDF5 file format) and are loaded for each value of the FF parameter. Sassena computes the selfincoherent intermediate structure factor, $I(Q, t)$, in the time-domain. To compare experiments and simulations we transform these data to the energy domain by using eq 2. i.e., $S_{\operatorname{sim}}(Q, E)$ which is commensurable to the experimental data $S_{\text {expt }}(Q, E)$. To simplify our calculations, the grid sizes, i.e., the energy bin width are adjusted to that of the experimental data. The values of $S_{\text {sim }}(Q, E)$ at the energy bins closest to $E \equiv 0$ are replaced with the adjacent bins. This step is necessary to remove the long-time dynamics that is not sampled by the simulation with sufficient statistical significance.

The resulting $S_{\text {sim }}(Q, E)$ is convoluted with the experimental resolution function as shown in eq 3. To achieve the same order of magnitude as the experimental data, the energy-integrated intensities are rescaled. The resulting fit optimizes the value of the FF parameter as well as the intensity constant for the first two terms ( $\alpha$ and $\beta$ ) and the linear background function ( $a$ and $b$ ).

The simplicity of this computational optimization technique relies on the workflow. The automated workflow performs vast majority of the work without manual intervention. The input parameter to be optimized is inserted in the system, and the workflow, as described in Fig. 1, performs the rest of the tasks. In the MD simulation scheme, the workflow automatically sends the computational jobs to a remote computer cluster, calculates the self-incoherent intermediate structure factor. The results are then transferred to a local workstation where the dynamic structure factor is calculated and is compared with the QENS data. The optimization of force field parameter is performed by matching the dynamic structure with the QENS data using an iterative procedure. The automatic workflow and the iterative procedures are repeated until the optimized parameter results into a force field parameter that is commensurate with QENS results. 


\section{Results: An example of the workflow}

We use the workflow described in the previous section to optimize a FF parameter of a fairly complex system consisting of nanodiamond (ND), transfer ribonucleic acid (tRNA) and water. The particular choice of this ND/tRNA/Water system is directed by the importance of RNA nanotechnology in the emerging field of nanomedicine, that can potentially be used to treat varieties of critical diseases such as, cancer, viral infection and genetic diseases [25]. Besides its electrical [26] and chemical [27] properties, the introduction of ND is guided by its low cytotoxicity and high biocompatibility [28] and hence can be used for in situ biological applications. Moreover, DNA has already been used to functionalize ND surface for biological applications [29] however, modification of ND surface with tRNA was never attempted before. Therefore, the choice of this particular example system is important to material design for future applications in nanomedicine.

Since the dynamics of tRNA in the ND/tRNA/Water system is crucial to design materials for future applications in nanomedicine [30], the dynamics of tRNA on the surface of a hydrophilic ND was studied using QENS and MD simulation [7]. QENS data were collected on the BASIS spectrometer [6] $(3.4 \mu \mathrm{eV}$ resolution in the $[-100,100] \mu \mathrm{eV}$ dynamic range) at temperatures $260 K, 280 K, 290 K$ and $300 K$. An additional run at temperature $14 K$ provided the resolution function. Water molecules were deuterated $\left(\mathrm{D}_{2} \mathrm{O}\right)$ instead of $\left.\mathrm{H}_{2} \mathrm{O}\right)$ to highlight the dynamics of tRNA. As the incoherent neutron scattering cross section of hydrogen is at least 40 times higher than any other atom in the sample, we captured only the dynamics of tRNA hydrogens. $S(Q, E)$ was binned along $Q$ into nine $Q$ slices and in $0.4 \mu \mathrm{eV}$ bins in the $[-100,100] \mu \mathrm{eV}$ range.

The models of the MD simulations were set up such that the model systems mimic the experimental conditions. The NAMD simulations were carried out with CHARMM27 [22] FF and the TIP3P [31] water model. The only caveat that hindered the accuracy of the MD simulation was the hydrophilic interactions between the carbon $(C)$ atom of the $\mathrm{ND}$ with the oxygen $(\mathrm{O})$ of the $\mathrm{D}_{2} \mathrm{O}$ molecule, as the FF parameter for this interaction was unknown. To simplify the problem, we used model LJ interactions between the $C$ of ND and $\mathrm{O}$ of $\mathrm{D}_{2} \mathrm{O}$. 
As discussed in the Section 1, hydrophilic interactions are typically modeled in two different ways, either incorporating explicit electrostatic forces between charged molecules or incorporating an attractive LJ interactions between the hydrophilic molecules. While the first method results in a FF with superior transferability properties, it is of-

ten difficult to find the right FF parameters and it is computationally expensive. Since we are interested in quantitatively reproduce the QENS measurements of a particular experiment, transferability is not a priority, accuracy is. Moreover, the accuracy in our simulation is guaranteed by the optimization of FF parameters. It is also important to note that the complex nature of ND surface jeopardizes the simulation setup in the first method since the ND surfaces are expected to be hydrophilic overall, but have mixed terminations, both purely hydrophilic and purely hydrophobic. Therefore, we opted for the second method by introducing attractive $\mathrm{LJ}$ interactions between ND and water and opted for $\epsilon_{C O}=-0.138 \mathrm{Kcal} / \mathrm{mol}$, between carbon and oxygen atoms following an earlier work representing attractive hydrophilic interactions [8]. The parameter of major concern, $\epsilon_{C O}$, is optimized as all the other interaction parameters of the simulation were accurately known.

We obtained the experimental $S(Q, E)$ profile data for the nine $Q$ slices and for four temperatures. The MD simulation data were generated from a system consisting of one ND, eight tRNA and water hydration level, $h=0.5$ for the equally spaced $Q$ values ranging from $0.3 \AA$ to $1.9 \AA$. While the experimental and simulation systems are analogous, experiments were performed with hydrated 'ND powder' instead of a single ND. Hence, the experiment contained more than one ND, thus we asserted the effect that putative interactions among ND particles may bear on measured tRNA dynamics. To resolve the discrepancy between experiment and simulation, we performed an extra set of MD simulations using eight ND and eight tRNA at a hydration level, $h=0.5$.

To calculate the $\epsilon_{C O}$ which best matches experimental QENS data, the SNS Pegasus workflow [17] used the NAMD and Sassena input files for the ND/tRNA/ $\mathrm{D}_{2} \mathrm{O}$ system. The simulations were performed for $10 \mathrm{~ns}$ each and the workflows used almost 400,000 CPU hours of time on a Cray XE6. The workflow automatically submitted nineteen parallel simulations for each temperature, scanning $\epsilon_{C O}$ from $-0.01 \mathrm{Kcal} / \mathrm{mol}$ to $-0.19 \mathrm{Kcal} / \mathrm{mol}$. The simulations were carried out on 800 cores with the equilibra- 


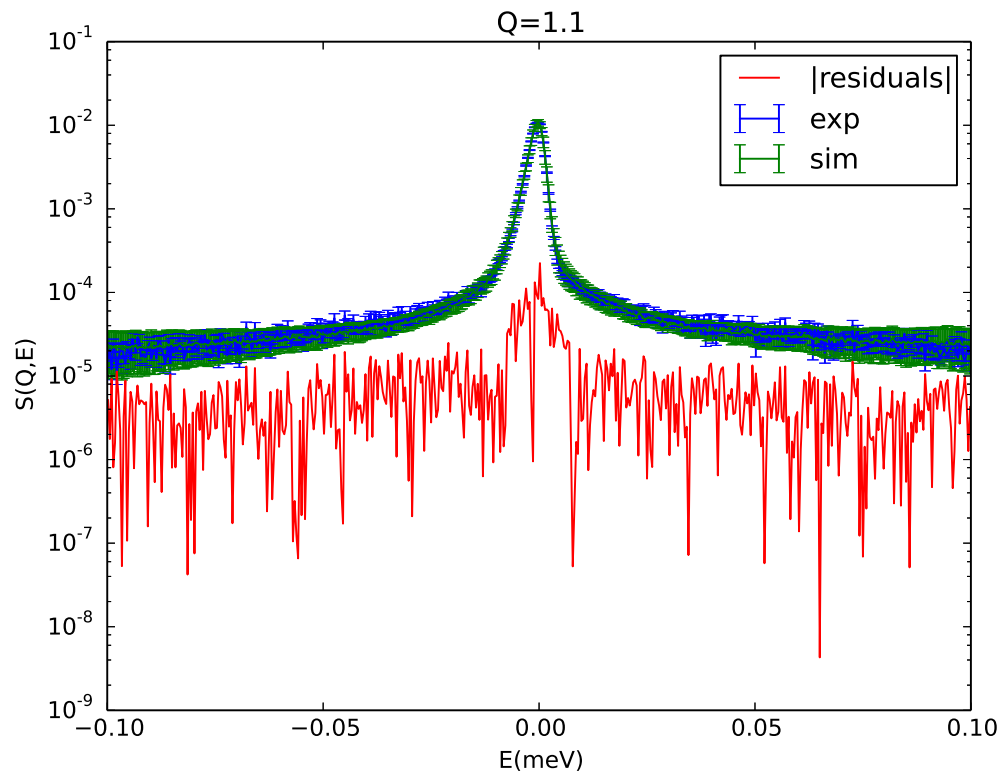

Figure 3: $S(Q, E)$ of the simulated ND/tRNA/Water system compared with experimental data for a midrange $Q$ that was used in the QENS experiment. Error bars for both experimental and simulation data are plotted

tion taking 1.5 hours and the production run taking 12-16 hours depending on the temperature. Once each NAMD job exits with a successful message code, the global translation and rotation are removed from the trajectory using a serial script available in AMBER [24] tool, cpptraj. The trajectories are then fed into Sassena to calculate QENS intensities. The Sassena jobs ran on 400 cores for 3-6 hours depending on the temperature and $Q$ values. When all the jobs from the remote section workflow are completed, they were transferred to a local workstation for optimization with in-house python tools [19].

The interpolation algorithm [19], DSFInterp, is called to create an interpolated structure factor in $\epsilon_{C O}$ using the nine $S\left(Q, E, f \equiv \epsilon_{C O}\right)$ profiles. In Fig. 3, we show the comparison between the $\epsilon_{C O}$-optimized $S(Q, E)$ with the experimental $S_{\text {expt }}(Q, E)$ for one of the $Q$ values. It can be seen from this figure that the optimization of $\epsilon_{C O}$ results in experimental and simulated $S(Q, E)$ profiles overlapping in all the dynamic range. Comparison for all nine values of $Q$ at $300 K$ are shown in Fig. 4 showing good 


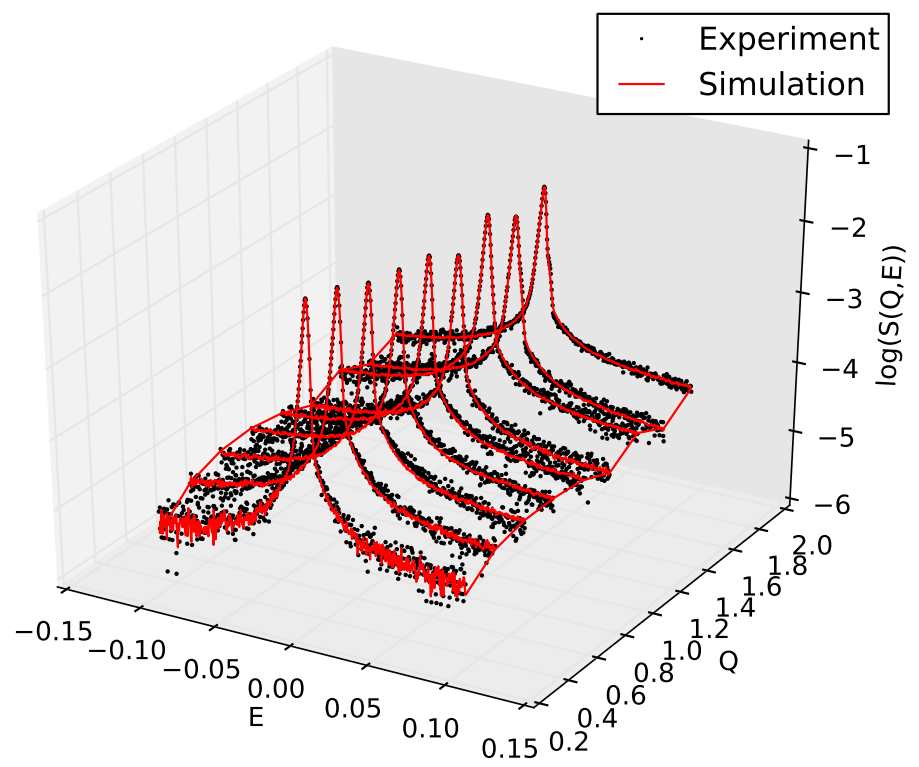

Figure 4: ND/tRNA/Water simulation with optimal value of $\epsilon_{C O}$ compared with experimental data points at all values of $Q$ at $300 K$ temperature. Natural logarithm of the structure factors, $S(Q, E)$, from the experiments and simulations at $300 \mathrm{~K}$ are plotted as a function of energy ( $E$ in the range $-0.15-+0.15$ ) and for scattering vector, $Q$, in the range $0.2-2.0$

match between experimental and optimized $S(Q, E)$. Thus, our simulated system can reproduce the dynamics of tRNA in the measured ranges of energy and momentum transfer, corresponding to $\approx 1 p s-1$ ss time range and $3 \AA$ to $20 \AA ̊$ length range.

In the MD simulation $\epsilon_{C O}$ was taken to be $-0.138 \mathrm{Kcal} / \mathrm{mol}$, the initial unoptimized FF. Therefore, in our optimization workflow, the reference value of $\epsilon_{C O}$ was $-0.138 \mathrm{Kcal} / \mathrm{mol}$. However, this is an approximate $\epsilon_{C O}$ as discussed earlier. The $\epsilon_{C O}=-0.138 \mathrm{Kcal} / \mathrm{mol}$ represent an attractive hydrophilic ND that closely resembles our system. The accurate value of the $\epsilon_{C O}$ is obtained by comparing the simulation data with the QENS data. The accuracies of the fits can be obtained from the goodness-of-fit shown in eq 4 , keeping $\epsilon_{C O}$ fixed. $\chi^{2}$ were calculated for each value of $Q$ and summed over for an overall accuracy of each interpolated value of $\epsilon_{C O}$. The $\epsilon_{C O}$-dependence of this cumulative $\chi^{2}$ (summed for all $Q$-slices) is shown in Fig. 5 (red squares) at $300 K$. The minimum of the interpolated $\chi^{2}$ yielded an optimized $\epsilon_{C O}=-0.1 \mathrm{Kcal} / \mathrm{mol}$. 


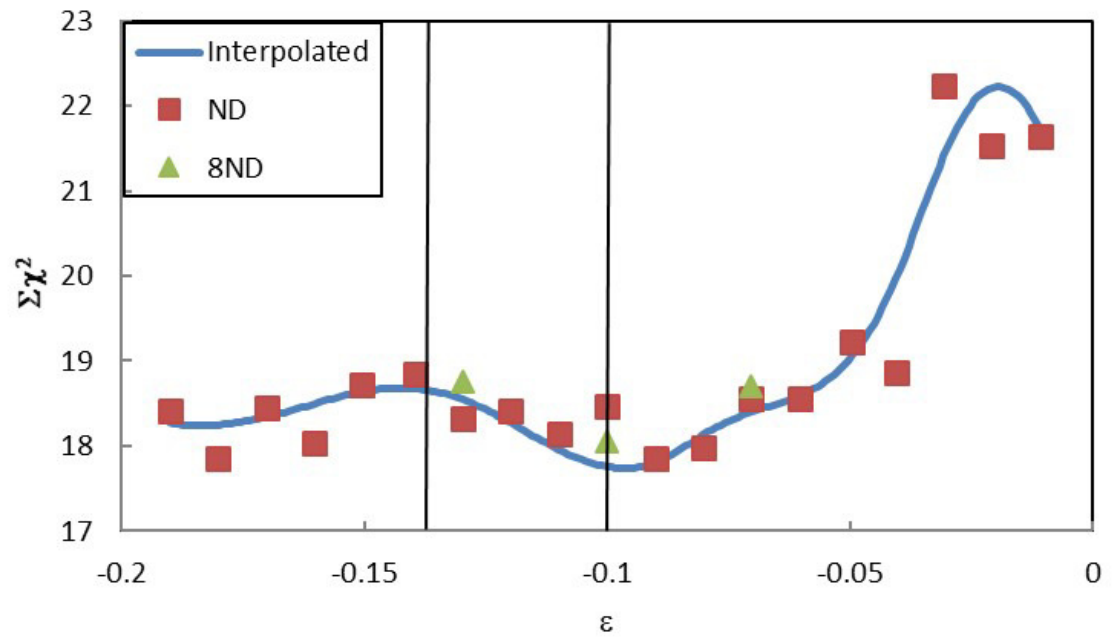

Figure 5: Optimization of $\epsilon$ for ND/tRNA/Water simulated molecule at $300 K$ showing both the interpolated values and $\chi^{2}$ for individual simulations representing the minimum value of $\epsilon_{C O}=0.1 \mathrm{Kcal} / \mathrm{mol}$. The left vertical line represents the 'guess' $\epsilon_{C O}=-0.138 \mathrm{Kcal} / \mathrm{mol}$ while the right vertical line represents the optimal value, $\epsilon_{C O}=-0.1 \mathrm{Kcal} / \mathrm{mol} . \chi^{2}$ is the sum of the squares of the residuals between experimental and computed structure factors weighted by the experimental error, also known as the goodness-of-fit. Green triangles show the more computationally expensive 8 nanodiamond model representing the minimum of $\epsilon_{C O}$ at $\epsilon_{C O}=0.1 \mathrm{Kcal} / \mathrm{mol}$.

Therefore, the optimized interaction parameter, $\epsilon_{C O}$, between ND and water is approximately $28 \%$ weaker than initially assumed in the MD simulation. The optimized $\epsilon_{C O}$ is accurate and a force field parameter for modeling the hydrophilic interaction of the ND surface in the MD simulation. We also performed three simulations for the eight ND model system at three different $\epsilon_{C O}$ values. The results (green triangle in Fig. 5) is approximately the same as the one ND $\chi^{2}$ values. We observed that the results were strikingly similar to the eight ND model. Therefore, it can be concluded that the surplus of ND particles, as it is found in ND powder in the experiment, will not have a significant effect on the overall tRNA dynamics.

Furthermore, optimization was also performed for three other temperatures, at $T=260 K, 280 K$, and $290 K$ for one ND system as shown in Fig. 6 The optimal $355 \epsilon_{C O}$ for all the temperatures lies around $\epsilon_{C O}=-0.1 \mathrm{Kcal} / \mathrm{mol}$ indicating no substan- 


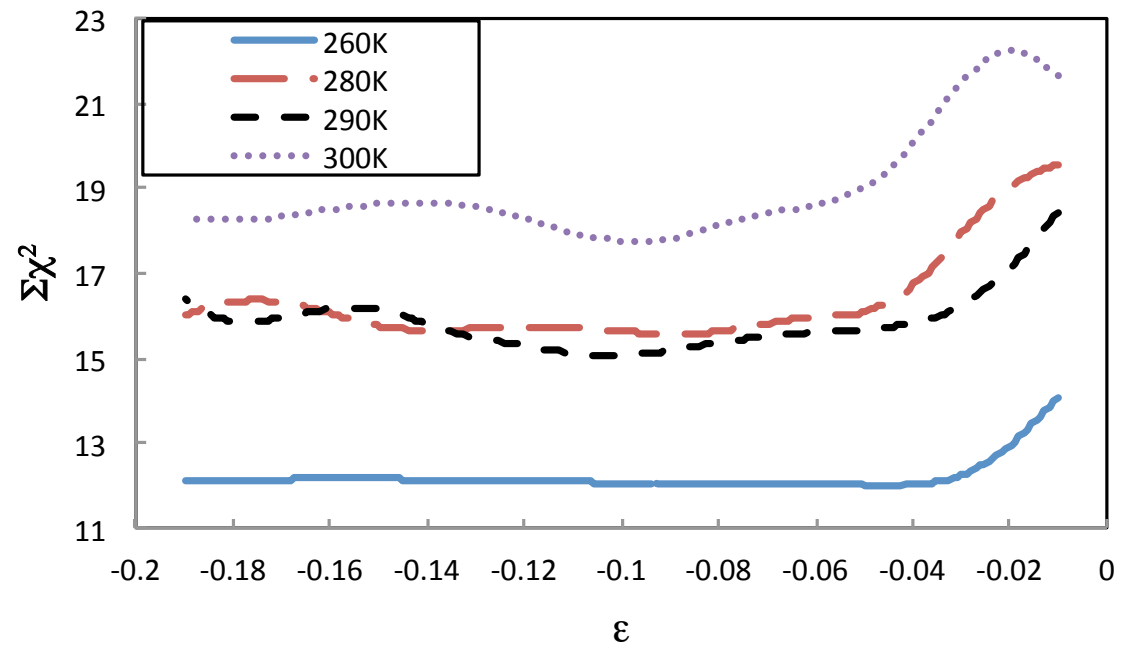

Figure 6: Optimization of $\epsilon$ for one ND/tRNA/Water simulated molecule at for temperatures, $260 \mathrm{~K}, 280 \mathrm{~K}$ and $290 K$ showing both the interpolated values and $\chi^{2}$ for individual simulations. All the temperatures show optimal $\epsilon_{C O}$ value close to the value found in $300 K$ representing the minimum value of $\epsilon_{C O}=-0.1$ $\mathrm{Kcal} / \mathrm{mol}$. For reference, $T=260 \mathrm{~K}$ (blue line) are shown although the optimization lacks accuracy. The lack of accuracy results from lack of achieving equilibrium below freezing temperature in the MD simulation.

tial changes in the optimized parameter with temperature. At $T=260 \mathrm{~K}$, inaccuracy in the optimization technique is observed resulting from a badly equilibrated MD simulation data below freezing temperature. This is shortcoming of the non-transferability of the FF, not an optimization issue. As the experiment was envisaged for applications in drug delivery system and, hence the temperature of importance is physiological temperature which is $300 K$. Therefore, we limited our discussion of the optimization technique for the physically sensible temperature, $T=300 \mathrm{~K}$. In an earlier work, we found the temperature dependence while optimizing the dipole moment of water in a concentrated solution of lithium chloride $(\mathrm{LiCl})[18]$ which is a consequence of the non-transferability of the FF. The temperature dependence of the optimal parameter is a shortcoming of the force-field, not an issue with our workflow.

It should be noted that in MD simulation, the fitted parameter, $\epsilon_{C O}$, is referred to as an energy parameter, but it is really just a force field parameter used in the calculation of the force between two particles. The interactions in MD simulations represent 
interaction between two individual particles. Temperature is a statistically averaged quantity and does not apply for individual particles. Hence, LJ interaction parameters do not depend on temperature for force calculations in MD simulations. Therefore, temperature dependence of the fitted LJ parameter (energy parameter) used in this simulation does not depend on temperature while performing the MD simulation.

\section{Conclusions}

We have developed a generalized workflow to optimize inaccurate FF parameters of MD simulations with neutron scattering data. As an example, we used the workflow to demonstrate the optimization technique using MD simulation and QENS data. We have incorporated resources available at SNS, such as Pegasus, Mantid project and NAMD simulation techniques into the generalized optimization technique. The workflow can run many simulations sampling the parameter space and then smoothens the results to find the global minima of the objective function. The simulations were run in parallel on a high performance computer at NERSC with the automated workflow managing the submission and processing of the results including moving the data to a local workstation for smoothing and optimization. Thus the workflow saves user time and reduces human error in manual submission of many different simulation runs. The workflow is easy to use and can be run with little knowledge of each of the software packages within the workflow.

We have tested this workflow for a complex system with ND, hydrated tRNA and water. Optimization with experimental data resulted in approximately $28 \%$ weaker interaction strength $\left(\epsilon_{C O}\right)$ between ND and water than originally assumed in the MD simulations. The weakening of the interactions allowed our MD simulations to quantitatively reproduce the enhanced mobility of hydrated tRNA when in the presence of ND, as observed from experimental QENS intensities [7]. The change in $\epsilon_{C O}$ will not affect the qualitative results of MD simulation, however for a quantitative comparison the optimized value of $\epsilon_{C O}$ must be used. We used this fitted $\epsilon_{C O}$ value to estimate the dynamical relaxation properties of the ND/tRNA/Water system and observed no substantial difference in the relaxation time, $\tau$ between $\epsilon_{C O}=-0.1 \mathrm{Kcal} / \mathrm{mol}$ and 
$\epsilon_{C O}=-0.138 \mathrm{Kcal} / \mathrm{mol}$. Therefore, the physical explanation of the observed enhanced dynamics of the tRNA in the presence of ND, as detailed in Dhindsa et al. [7], remains unchanged by using the optimized value of $\epsilon_{C O}=0.1 \mathrm{Kcal} / \mathrm{mol}$. This workflow is an important addition to the neutron experimental community and can be accessed by users performing neutron experiments at SNS. While we tested the workflow for QENS experimental data, it can be generalized to other neutron experiments such as, small angle neutron scattering (SANS) and spin echo, or in combination with data from light or X-ray sources.

\section{Acknowledgements}

The development workflow was supported by the US Department of Energy (DOE), Office of Science, Basic Energy Sciences (BES), Materials Sciences and Engineering Division. The use of Oak Ridge National Laboratory's (ORNL) SNS was sponsored by the Scientific User Facilities Division, Office of BES. We thank Gurpreet K. Dhindsa and Xiang-Qiang Chu from Wayne State University for providing us with the QENS data. The Pegasus workflow was funded by the DOE under contract number DE-SC0012636. This manuscript has been authored by ORNL, which is managed by UT- Battelle, LLC under Contract No. DE-AC05-00OR22725 with the U.S. DOE. This product includes software developed by and/or derived from the Globus project (http://www.globus.org/). This research used resources of the National Energy Research Scientific Computing Center (NERSC), a DOE Office of Scientific User Facility supported by the Office of Science of the U.S. DOE under Contract No. DE-AC0205CH11231. The original NAMD simulations were performed at TITAN supercomputer at the National Center for Computational Sciences and used resources of the Oak

Ridge Leadership Computing Facility at the ORNL, which is supported by the Office of Science of the U.S. DOE under Contract No. DE-AC05-00OR22725. Part of this research was conducted at the Center for Nanophase Materials Sciences (CNMS), which is a DOE Science User Facility. 


\section{References}

[1] Spallation neutron source I neutron science at ornl, accessed: August 09, 2016. URL https://neutrons.ornl.gov/sns

[2] D. Bhowmik, N. Malikova, G. Meriguet, O. Bernard, J. Teixeira, P. Turq, Aqueous solutions of tetraalkylammonium halides: ion hydration dynamics and ionion interactions in light of steric effects, Phys. Chem. Chem. Phys. 16 (2014) 13447.

[3] H. Zhang, S. Khodadadi, S. L. Fiedler, J. E. Curtis, Role of water and ions on the dynamical transition of rna, J. Phys. Chem. Lett. 4 (2013) 3325.

[4] J. Heyda, T. Hrobarik, P. Jungwirth, Ion-specific interactions between halides and basic amino acids in water, J. Phys. Chem. A 113 (2009) 1969.

[5] L. Garcia-Tarres, E. Guardia, Hydration and dynamics of a tetramethylammonium ion in water: A computer simulation study, J. Phys. Chem. B 102 (1998) 7448.

[6] E. Mamontov, K. W. Herwig, A time-of-flight backscattering spectrometer at the spallation neutron source, basis, Rev. Sci. Instrum. 82 (2011) 085109.

[7] G. K. Dhindsa, D. Bhowmik, M. Goswami, H. O’Neil, E. Mamontov, B. G. Sumpter, L. Hong, P. Ganesh, X. q. Chu, Enhanced dynamics of hydrated trna on nanodiamond surfaces: A combined neutron scattering and md simulation study, J. Phys. Chem. B 120 (2016) 10059.

[8] C. Sendner, D. Horinek, L. Bocquet, R. R. Netz, Interfacial water at hydrophobic and hydrophilic surfaces: slip, viscosity, and diffusion, Langmuir 25 (2009) 10768.

[9] L. Garcia-Tarres, E. Guardia, Destructive extraction of phospholipids from escheria coli membranes by graphene nanosheets, Nat. Nanotechnol. 8 (2013) 594.

[10] P. G. de Gennes, Collective motion of hydrogen bonds, Solid State Commun. 1 (1963) 132. 
[11] M. Bee, Quasielastic Neutron Scattering, Principles and Applications in Solid State Chemistry, Biology and Materials Science, Taylor \& Francis, 1988. management system for science automation, Future Generation Computer Systems 46 (2015) 17-35. doi:10.1016/j.future.2014.10.008.

1

[12] P. Schofield, Space-time correlation function formalism for slow neutron scattering, Phys. Rev. Lett. 4 (1960) 239.

[13] E. Deelman, C. Carothers, A. Mandal, B. Tierney, J. S. Vetter, I. Baldin, C. Castillo, G. Juve, D. Król, V. Lynch, B. Mayer, J. Meredith, T. Proffen, P. Ruth, R. F. da Silva, Panorama: An approach to performance modeling and diagnosis of extreme-scale workflows Int. J. High Perform. Comput. Appl. (2012) doi:10.1177/1094342015594515 URL http://dx.doi.org/10.1177/1094342015594515

[14] J. C. Phillips, R. Braun, W. Wang, J. Gumbart, E. Tajkhorshid, E. Villa, C. Chipot, R. D. Skeel, L. Kale, K. Schulten, Scalable molecular dynamics with namd, J. Comput. Chem. 26 (2005) 1781.

[15] B. Lindner, J. C. Smith, Sassena - x-ray and neutron scattering calculated from molecular dynamics trajectories using massively parallel computers, Comp. Phys. Comm. 183 (2012) 9.

[16] O. Arnold, J. Bilheux, J. Borreguero, A. Buts, S. Campbell, L. Chapon, M. Doucet, N. Draper, R. F. Leal, M. Gigg, V. Lynch, A. Markvardsen, D. Mikkelson, R. Mikkelson, R. Miller, K. Palmen, P. Parker, G. Passos, T. Perring, P. Peterson, S. Ren, M. Reuter, A. Savici, J. Taylor, R. Taylor, R. Tolchenov, W. Zhou, J. Zikovsky, MantidâǍŤdata analysis and visualization package for neutron scattering and $\mu$ sr experiments, Nucl. Instr. Meth. Phys. Res., A 764 (2014) 156.

[17] E. Deelman, K. Vahi, G. Juve, M. Rynge, S. Callaghan, P. J. Maechling, R. Mayani, W. Chen, R. L. F. da Silva, M. Livny, K.Wenger, Pegasus: a workflow URL http://pegasus.isi.edu/publications/2014/ 2014-fgcs-deelman.pdf 
[18] J. Borreguero, S. I. Campbell, O. A. Delaire, M. Doucet, M. Goswami, M. E. Hagen, V. E. Lynch, T. E. Proffen, S. Ren, A. T. Savici, B. G. Sumpter, Integrating advanced materials simulation techniques into an automated data analysis workflow at the spallation neutron source, in: TMS 2014 Supplemental Proceedings, John Wiley \& Sons Inc., Hoboken, New Jersey, USA, 2014, p. 297.

[19] V. E. L. J. M. Borreguero, Molecular dynamics force-field refinement against quasi elastic neutron scattering data, J. Chem. Theory Comput. 12 (2016) 9.

[20] Htcondor manual, version 8.2.10.

nURL https://research.cs.wisc.edu/htcondor/manual/v8.2/ ref.html

[21] W. D. Cornell, P. Cieplak, C. I. Gould, J. K. M. Merz, D. M. Ferguson, D. C. Spellmeyer, T. Fox, J. W. Caldwell, P. A. Kollman, A second generation force field for the simulation of proteins, nucleic acids, and organic molecules, J. Am. Chem. Soc. 117 (1995) 5179.

[22] B. R. Brooks, C. L. B. III, A. D. M. Jr, L. Nilsson, R. J. Petrella, B. Roux, Y. Won, G. Archontis, C. Bartels, S. Boresch, A. Caflisch, L. Caves, Q. Cui, A. Dinner, M. Feig, S. Fischer, J. Gao, M. Hodoscek, W. Im, K. Kuczera, T. Lazaridis, J. Ma, V. Ovchinnikov, E. Paci, R. Pastor, C. Post, J. Pu, M. Schaefer, B. Tidor, R. M. Venable, H. L. Woodcock, X. Wu, W. Yang, D. York, M. Karplus, Charmm: The biomolecular simulation program, J. Comput. Chem. 30 (2009) 1545.

[23] A. T. Brunger, P. D. Adams, Molecular dynamics applied to X-ray structure refinement, Acc. Chem. Res. 35 (2002) 404.

[24] D. A. Case, R. Betz, W. Botello-Smith, D. Cerutti, I. T.E. Cheatham, T. Darden, R. Duke, T. Giese, H. Gohlke, A. Goetz, N. Homeyer, S. Izadi, P. Janowski, J. Kaus, A. Kovalenko, T. Lee, S. LeGrand, P. Li, C. Lin, T. Luchko, R. Luo, B. Madej, D. Mermelstein, K. Merz, G. Monard, H. Nguyen, H. Nguyen, I. Omelyan, A. Onufriev, D. Roe, A. Roitberg, C. Sagui, C. Simmerling, J. Swails, R. Walker, J. Wang, R. Wolf, X. Wu, L. Xiao, D. York, P. Kollman, AMBER 15, University of California, San Fransico, CA, 2015. 
[25] Puo, The emerging field of rna nanotechnology, Nat. Nanotechnol. 5 (2010) 833.

[26] G. M. Swain, M. Ramesham, The electrochemical activity of boron-doped polycrystalline diamond thin-film electrodes, Anal. Chem. 65 (1993) 345.

[27] V. N. Mochalin, O. Shenderova, D. Ho, Y. Gogotsi, The properties and applications of nanodiamond, Nat. Nanotechnol. 7 (2012) 11.

[28] V. N. Mochalin, A. Pentecost, X.-M. Li, I. Nietzel, M. Nelson, C. Wei, T. He, F. Guo, Y. Gogotsi, J. H.-T. Park, Adsorption of drugs on nanodiamond: Towards development of a drug delivery platform, Mol. Pharm. 10 (2013) 3728.

[29] W. Yang, O. Auciello, J. E. Butler, W. Cai, J. A. Carlisle, J. E. Gerbi, D. M. Gruen, T. Knickerbocker, T. L. Lasseter, J. N. R. Jr., L. M. Smith, R. J. Hamers, Dna-modified nanocrystalline diamond thin-film as stable, biologically active substrates, Nat. Mater. 1 (2002) 253.

[30] S. Pronk, E. Lindahl, P. M. Kasson, Dynamic heterogeneity controls diffusion and viscosity near biological interfaces, Nat. Commun. 5 (2014) 3034.

525 [31] W. L. Jorgensen, J. Chandrasekhar, J. D. Madura, R. W. Impey, M. L. Klein, Comparison of simple potential functions for simulating liquid water, J. Chem. Phys. 79 (1983) 926. 


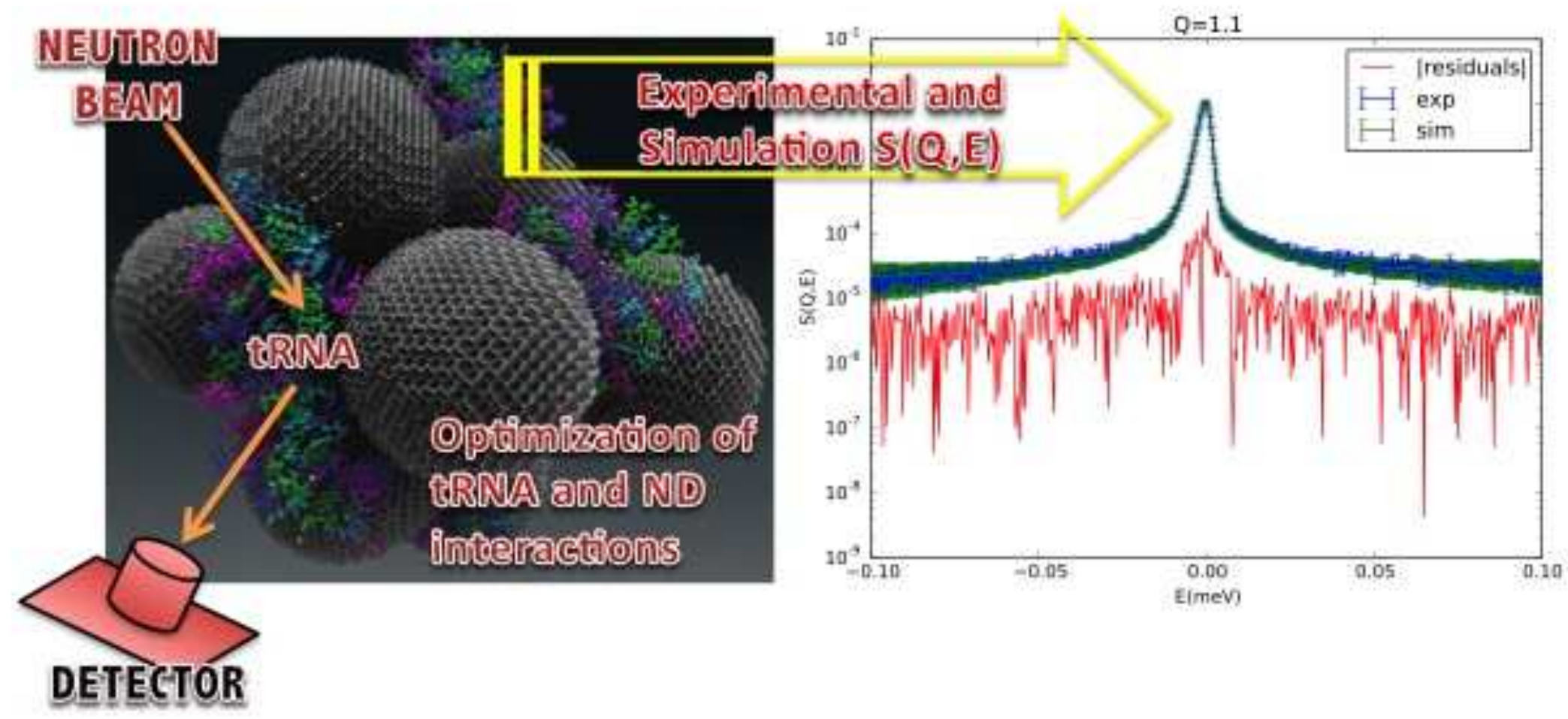

